\title{
Radiative type-III ELMy H-mode in all-tungsten ASDEX Upgrade
}

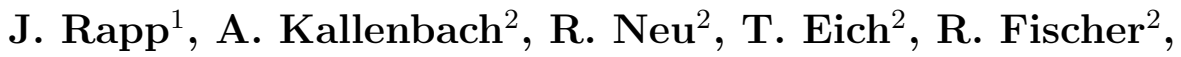 \\ A. Herrmann ${ }^{2}$, S. Potzel ${ }^{2}$, G.J. van Rooij ${ }^{3}$, J.J. Zielinski ${ }^{3}$ and \\ ASDEX Upgrade team \\ 1 Oak Ridge National Laboratory, Oak Ridge, Tennessee, USA \\ 2 Max-Planck-Institut für Plasmaphysik, EURATOM Assoziation, Garching, \\ Germany \\ 3 Dutch Institute For Fundamental Energy Research, EURATOM Association, \\ Trilateral Euregio Cluster, Nieuwegein, The Netherlands
}

\begin{abstract}
The type-III ELMy H-mode might be the solution for an integrated ITER operation scenario fulfilling the fusion power amplification factor (output fusion power to input heating power) of $Q=10$ with simultaneous acceptable steady-state and transient power loads to the plasma facing components. This highly radiative type-III ELMy H-mode is achieved by nitrogen seeding. Experiments on the tokamak ASDEX Upgrade, in which all plasma facing components are coated with tungsten showed favourable confinement conditions $\left(H_{98(y, 2)}=1\right)$ at high plasma pressure $\left(\beta_{N}=2.4\right)$. The power load to the plasma facing components is as low as $\approx 3 \mathrm{MW} / \mathrm{m}^{2}$ during the peak heat loads due to Edge Localized Modes (ELMs) at high radiative power fractions of $f_{\text {rad }} \approx 0.75$. In those high density discharges the central impurity concentration is very low as a result of hollow nitrogen density profiles and minimal erosion of tungsten. The tungsten erosion is not only suppressed in-between ELMs, when the divertor is detached, but also during the type-III ELM activity. Such low impurity concentrations in the plasma core might lead to even higher fusion amplification factors in ITER than 10. This is a demonstration of the compatibility of the radiating type-III ELMy Hmode with a tungsten divertor and main chamber wall, with sufficient confinement and favourable power exhaust characteristics. Together with previous results in all-carbon devices this demonstration strengthens the case for a potential application of a $Q=10$ scenario on ITER.
\end{abstract}

PACS numbers: 52.25.Vy, 52.40.Hf, 52.55.Fa, 52.55Rk 
For future fusion reactors like ITER, which is currently under construction in Southern France, an operation regime which combines good confinement and acceptable power load to the plasma facing materials is the ultimate challenge. In order to reduce the steady-state power flux to the plasma facing components in the divertor to technological managable levels of $10 \mathrm{MW} / \mathrm{m}^{2}$ the conducted peak heat flux has to be dissipated by impurity radiation. This is achieved by seeding gaseous impurities like argon, neon or nitrogen. The electrons are cooled by electron impact with the impurities and line-radiation in particular of the Be- and Li-like impurity ions exhausts the thermal power. In addition instabilities in the edge plasma of a tokamak in the high confinement mode (H-mode) [1] lead to transient heat loads to the plasma facing components. The power load due to these so-called Edge Localized Modes (ELMs) [2] are one of the most significant obstacles on the way to a fusion reactor $[3,4]$. Several mitigation schemes have been proposed [5, 6, 7]. All of them have their drawbacks because they either lead to reduced confinement or they exist only in a very narrow operational domain. All of them do not combine the requirements of power exhaust and good confinement as required for ITER. Since large ELMs as they are expected to occur in ITER, will most likely not be dissipated by impurity radiation [8], the type-III ELMy $\mathrm{H}$-mode is proposed as an alternative ITER scenario [9], which has naturally smaller ELMs. Although those type-IIIs are most likely acceptable with respect to the power fluxes to the plasma facing components, the lower confinement in this operating regime will make it necessary for ITER to operate at its absolute maximum plasma current of 17MA. Recent experiments at JET, which have been extrapolated to ITER, have resulted in the hope that this regime might be able to fulfill even the conditions of the ITER 15MA scenario [10]. Access to the type-III ELMy H-mode was demonstrated in a large operation domain, down to a collisionality of $\nu^{*}=0.045$ for impurity seeded and even less $\left(\nu^{*}=0.023\right)$ for unseeded discharges $[10,11]$.

An important prerequisite for this scenario is the strong deuterium fuelling to achieve high plasma densities close to the Greenwald limit [12]. The high density operation has also the advantage that the impurity levels in the plasma core of the fusion plasma are kept low [18], which is important for maximizing the fusion power in the core. All the experiments reported above were carried out in all-carbon devices. Carbon as plasma facing material is a forgiving material, but has the disadvantage of forming hydrocarbons during the erosion process, which might migrate to remote areas leading to hydrogen rich carbon layers. Those hydrogen rich carbon layers could cause a tritium inventory problem in a fusion reactor, if they are not accessible for cleaning and hence the tritium is irrecoverable. Due to this problem ITER will be a metallic device with plasma facing components made of Be and $\mathrm{W}$. This will pose a new problem for radiative scenarios, since carbon is not available as a radiative impurity and thus more extrinsic impurities have to be used to reach the required radiative power fractions.

In order to test the radiative type-III ELMy H-mode in an all metal environment experiments were carried out on ASDEX Upgrade. ASDEX Upgrade a divertor tokamak $(R=1.65 \mathrm{~m}, a=0.5 \mathrm{~m})[13]$ is an all-tungsten device, meaning that all plasma facing 
components (mostly graphite) have a tungsten coating [14]. In the experiments reported here nitrogen was used as an extrinsic impurity (see also [15, 16]). Nitrogen has the advantage of radiating efficiently at low electron temperatures. Thus the radiation zone from nitrogen is mostly located in the divertor region. A good comparison of divertor to bulk radiation for different seeded impurities has been carried out on JET [17], which confirmed that nitrogen is the best impurity to limit the impurity radiation to the divertor region at high radiative power fractions. On ASDEX Upgrade the discharges were fuelled with deuterium and nitrogen with rates up to $4 \times 10^{22} \mathrm{el} / \mathrm{s}$ feed forward to keep the density and radiative power fraction constant during the auxiliary heating period. The discharges were heated by neutral beam injection (NBI) with power steps up to $12 \mathrm{MW}$. In addition $1 \mathrm{MW}$ ion cyclotron resonance heating (ICRH) was used to prevent impurity accumulation in the plasma core. This is a typical method used in impurity seeded discharges [19, 20]. A typical discharge is shown in figure 1.

The type-III ELM regime was identified by the ELM frequency reduction during the power steps in the discharge as well as by the MHD signature of the type-III ELMs. The radiative power in those type-III ELMy H-modes at $1.2 \mathrm{MA} / 2.5 \mathrm{~T}\left(q_{95}=3.9\right)$ and $1.2 \mathrm{MA} / 2.0 \mathrm{~T}\left(q_{95}=3.1\right)$ was more than $70 \%$. The electron density is $80 \%$ of the Greenwald density in the low triangularity pulses, although the absolute density is very high $\left(\bar{n}_{e}=1.2 \times 10^{20} \mathrm{~m}^{-3}\right)$, as a matter of fact $20 \%$ higher than foreseen in ITER. For both edge safety factors $\left(q_{95}\right)$ a power scan was carried out. It was possible to reach a maximum pressure of $\beta_{N}=2.4$ at the end of the power steps (see figure 1). Increasing the power in those nitrogen seeded type-III ELMy H-mode discharges leads to an increase of the confinement similar as in type-I ELMy H-modes [16] resulting in a confinement enhancement factor $H_{98(y, 2)}$ as high as 1 at the highest plasma pressure (see figure 2). Only at the end of the discharge after the rollover in confinement and $\beta_{N}$ (possibly an indication of a $\beta$-limit), the plasma density peaks and leads to an impurity accumulation further amplifying the confinement loss. The radiated power fraction reaches almost $100 \%$ at the end of the discharge (see figure 1). Utilizing feedback on the central heating power as well as on the gas fuelling is necessary to stabilize the discharge at high $\beta_{N}$. It should be mentioned that the increase of the confinement with the heating power is similar to the improved H-mode or Hybrid scenario. However, in the type-III ELMy H-mode no efforts to the tailoring and optimizing of the q-profile have been spent.

The type-III ELM frequencey in these discharges varies between 150 and $500 \mathrm{~Hz}$, which is very similar to measurements on JET [10]. The power flux density during the ELMs was reduced to less than $4 \mathrm{MW} / \mathrm{m}^{2}$ (see figure 3). At these low power flux densities and high divertor densities the tungsten erosion in the divertor is suppressed to negligible values. The tungsten influx from the divertor is dominated by ELMs. Figure 4 shows the tungsten influx from the divertor during the ELM peak. Since the physical sputtering yield of tungsten, as a results of impinging light impurities, is strongly reduced at electron temperatures below $10 \mathrm{eV}$, it is not surprising that the tungsten influx is reduced significantly. The comparison of the $\mathrm{W}$ influx for type-III 
ELMy H-modes with type-I ELMy H-modes shows a suppression of the tungsten influx of about an order of magnitude in the type-III ELMy H-modes. For the time-averaged $\mathrm{W}$ influx this reduction is in the same range.

Of course it is important to know how the power fluxes in these radiative type-III ELMy H-modes scale to ITER. For the type-I ELMs a multi-machine scaling has been developed [4], in which the ELM energy loss normalized to the pedestal stored energy loss could be best mapped versus the pedestal collisionality. In figure 3 the peak power flux density is plotted versus the collisionality, which does not show any dependence on the collisionality. It appears that the power decay length on the target during the type-III ELM activity is slightly decreasing (by about 30\%), whereas the power decay time is increasing from about $1 \mathrm{~ms}$ at $\nu^{*}=0.9$ to about $2 \mathrm{~ms}$ at $\nu^{*}=0.4$. The ELM rise time is constant at about $0.5 \mathrm{~ms}$ over the collisionality range investigated. The increase of the power decay time towards lower collisionality is most likely due to the nature of the type-III ELM instability, which is thought to be linked to a critical edge plasma current gradient. Lower collisionality means lower resistivity. Hence the time required to alter the current profile in the plasma edge is longer at lower collisionality and thus the duration of the instability becomes longer.

The ELM energy loss normalized to the total stored energy is even dropping towards lower collisionality. This is similar to the findings on JET, where the energy loss due to type-III ELMs in nitrogen seeded discharges is reduced slightly torwards lower collisionalities [10]. In figure 5 both the data from JET and ASDEX Upgrade are shown. In both tokamaks at a collisionality of $\nu^{*}=0.5$ the ELM energy loss is about $0.1 \%$ of the total stored energy and is decreasing towards lower collisionality of $\nu^{*}=0.25$. An extrapolation of this trend to ITER collisionalities $\nu^{*}=0.01$ for the standard $15 \mathrm{MA}$ scenario and $\nu^{*}=0.04$ for the high density $17 \mathrm{MA}$ scenario [10] is very difficult to make over one order of magnitude. However, even if the data shown in figure 5 are not extrapolated towards the ITER collisionality (with decreasing ELM energy loss towards lower collisionality), the data shown here reinforce the simple predictions made for ITER [10], which lead to the conclusion that the expected divertor power load due to type-III ELMs with $\Delta W / W \approx 0.1 \%$ in ITER is approximately $0.1 \mathrm{MJ} / \mathrm{m}^{2}$ [10]. This is within the allowed level of acceptance for ITER.

The pollution of the plasma is very low. It appears that the $Z_{\text {eff }}$ profile is hollow in these nitrogen seeded discharges. This is similar to observations at JET, where the nitrogen concentration profile in the plasma center was found to be hollow [5]. In addition, as already stated above (see also figure 4), the erosion of tungsten is strongly suppressed in these nitrogen seeded type-III ELMy H-modes (see also [21]). The $Z_{\text {eff }}$ values are compared to scaling laws. The scaling laws typically have a relation between the radiated power, the electron density and the impurity concentration in the plasma. Recently an updated $Z_{\text {eff }}$ scaling law, which takes into account the confinement time, $Z_{\text {eff }}=1+40 P_{\text {rad }} Z^{0.12} \tau_{E} S^{-0.94} n_{e}^{-1.5} a_{m i n}^{-1} R^{-1}$, has been compared to experimental data from JET [18]. Since the $Z_{\text {eff }}$ profiles are hollow and in some cases invalid in the plasma center (due to ECE cut-off at those high densities), for the comparison the $Z_{\text {eff }}$ values 
at the plasma edge were taken, as this is where most of the plasma radiation originates. It should be noted that this is usually not done, and that the $Z_{\text {eff }}$ values in these comparisons are mostly line-averaged data. Figure 6 shows this comparison. It appears that within the error bars of the measurements (25\%) the experimental values are in agreement with this scaling.

In summary these experiments in the all-tungsten ASDEX Upgrade confirm the potential of this highly radiating type-III ELMy H-mode by nitrogen seeding to be a strong contender as THE operation scenario for ITER.

\section{Acknowledgments}

Part of this work was financially supported by the Nederlandse Organisatie voor Wetenschappelijk Onderzoek (NWO). It is supported by the European Communities under the contract of Association between EURATOM and FOM and carried out within the framework of the European Fusion Programme.

\section{References}

[1] F. Wagner et al. 1982 Phys. Rev. Lett. 191408

[2] H. Zohm et al. 1996 Plasma Phys. Control. Fusion 38105

[3] G. Federici et al. 2003 Plasma Phys. Control. Fusion 451523

[4] A. Loarte et al. 2003 J. Nucl. Mater. 313-316 919

[5] J. Rapp et al. 2002 Plasma Phys. Control. Fusion 44639

[6] T.E. Evans et al. 2004 Phys. Rev. Lett. 92235003

[7] P.T. Lang et al. 2004 Nucl. Fusion 44665

[8] J. Rapp et al. 2004 Nucl. Fusion 44312

[9] J. Rapp et al. 2005 J. Nucl. Mater. 337-339 826

[10] J. Rapp et al. 2009 Nucl. Fusion 49095012

[11] M. Valovic et al. 2004 Plasma Phys. Control. Fusion 461877

[12] M. Greenwald et al. 1988 Nucl. Fusion 282199

[13] A. Herrmann, O. Gruber 2003 Fus. Sci. Technol. 44569

[14] R. Neu et al. 2007 Plasma Phys. Control. Fusion 49 B59

[15] A. Kallenbach et al. 2010 Plasma Phys. Control. Fusion 52055002

[16] J. Schweinzer et al. 2011 Nucl. Fusion 51113003

[17] L.C. Ingesson et al. 2003 J. Nucl. Mater. 313-316 1173

[18] J. Rapp et al. 2009 J. Nucl. Mater. 390-391 238

[19] M.F.F. Nave et al. 2003 Nucl. Fusion 431204

[20] R. Neu et al. 2003 J. Nucl. Mater. 313-316 116

[21] R. Neu et al. 2010 J. Nucl. Mater. 415 S322 


\section{Figure captions}

Figure 1. Overview of nitrogen seeded type-III ELMy H-mode on Asdex Upgrade. Neutral Beam Heating power (black), ICRH heating power (green), total heating power (black), radiated power (red); line-averaged electron density; Greenwald density fraction; beta normalized; confinement enhancement factor $H_{98(y, 2)} ; H_{\alpha}$ measurements in divertor.

Figure 2. Confinement enhancement factor $H_{98(y, 2)}$ versus normalized $\beta_{N}$.

Figure 3. Maximum power flux during type-III ELMs in the outer divertor.

Figure 4. Tungsten source in the divertor as derived from the WI line emission (400.8nm) during the ELM peak as a function of the divertor electron temperature: comparison of nitrogen seeded type-III ELMy H-modes with nitrogen seeded type-I ELMy H-modes for similar separatrix densities $\left(n_{e} \approx 3.1 \times 10^{19} \mathrm{~m}^{-3}\right)$.

Figure 5. ELM energy loss normalized to plasma stored energy, derived from IR thermography in outer divertor, comparison of radiative, nitrogen fuelled, type-III ELMy H-modes in JET and ASDEX Upgrade.

Figure 6. $Z_{\text {eff }}$ values measured in ASDEX Upgrade compared with empirical scaling. 

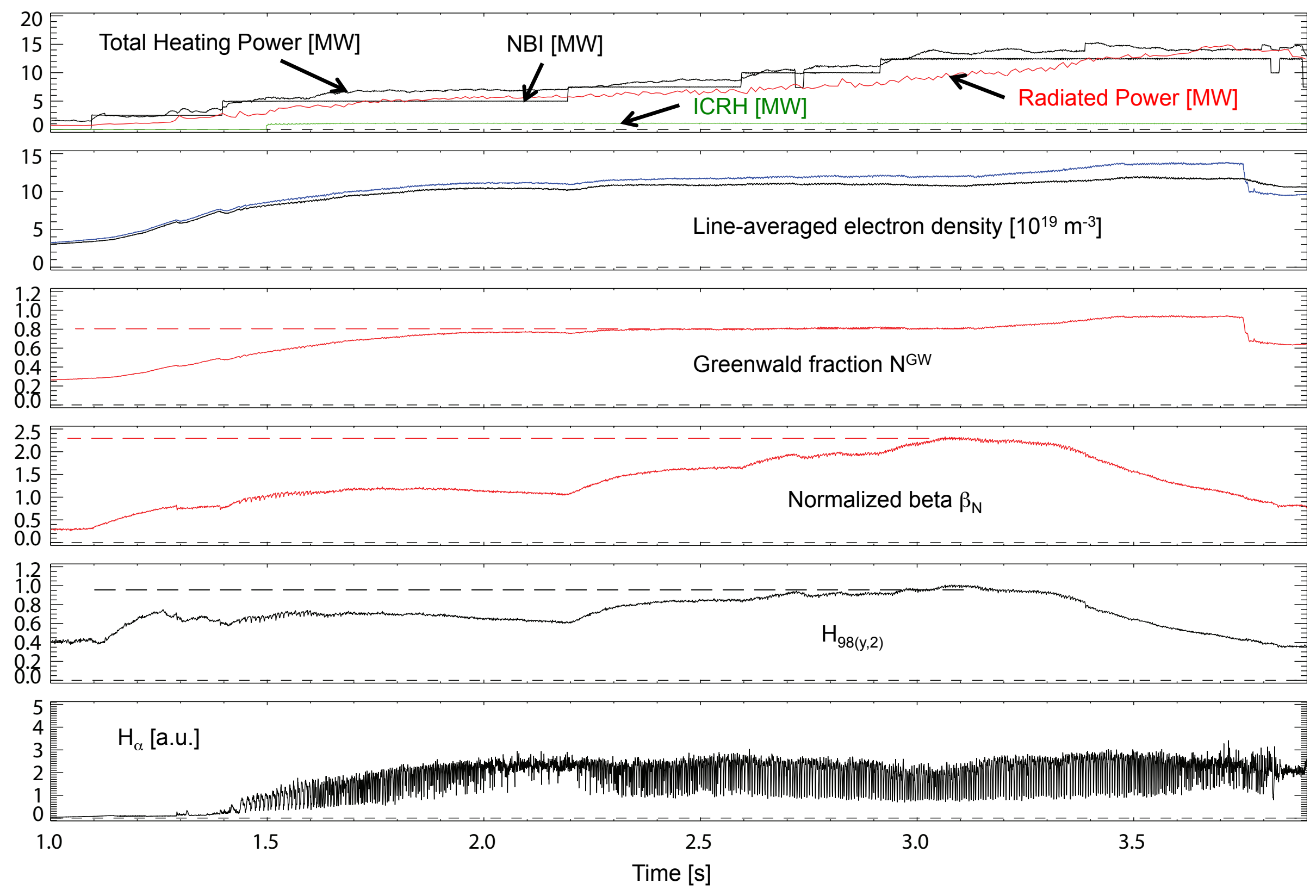


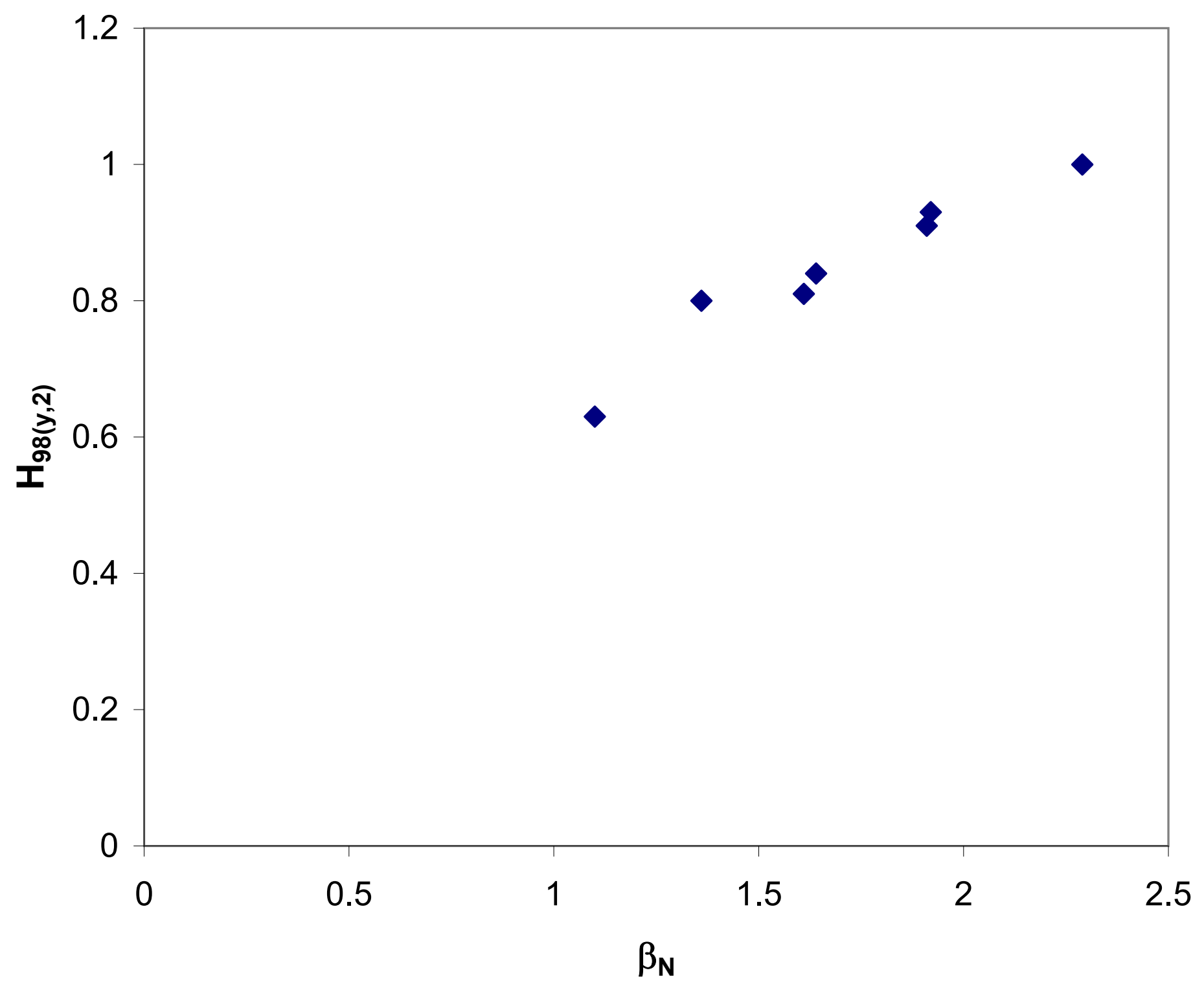




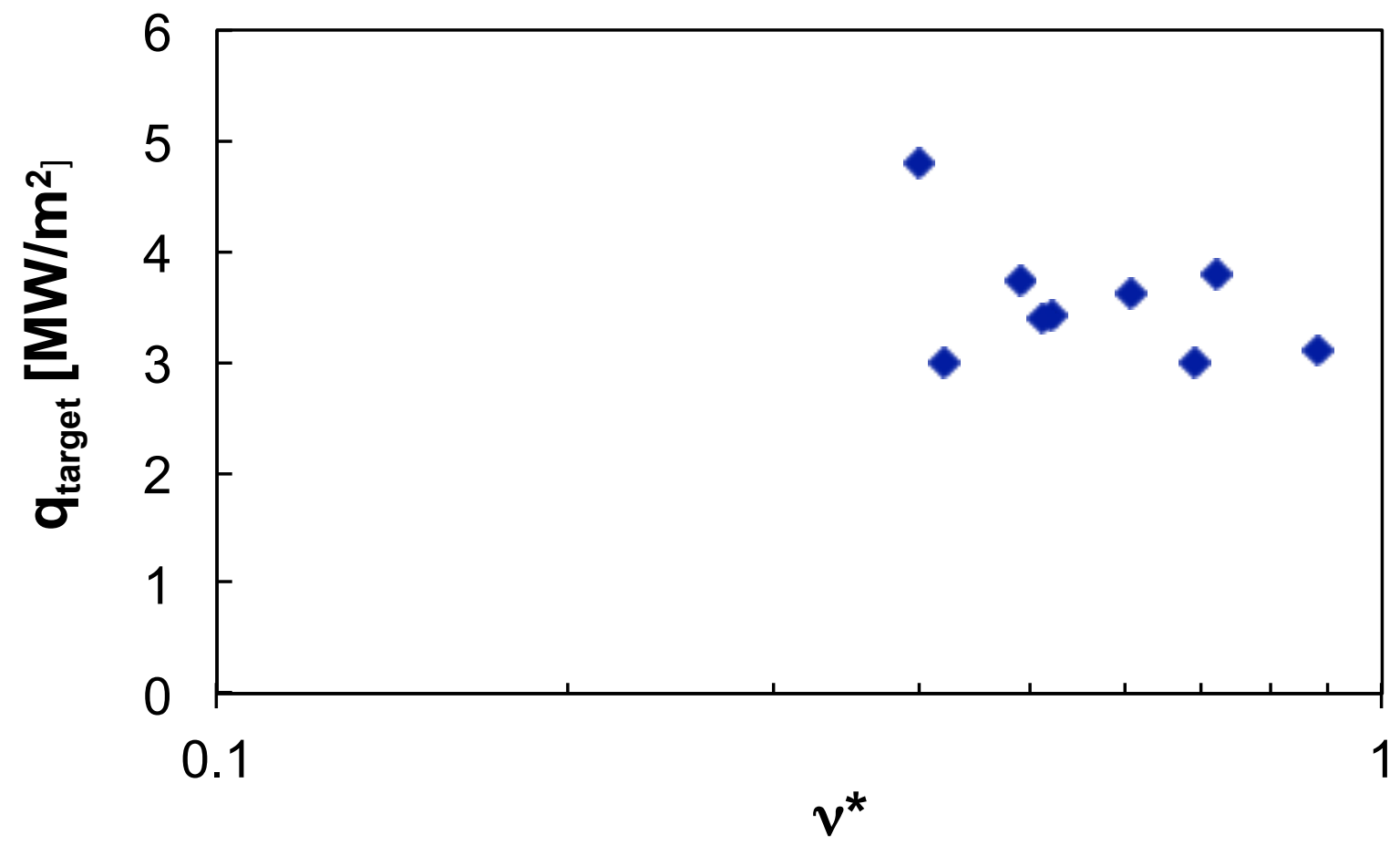




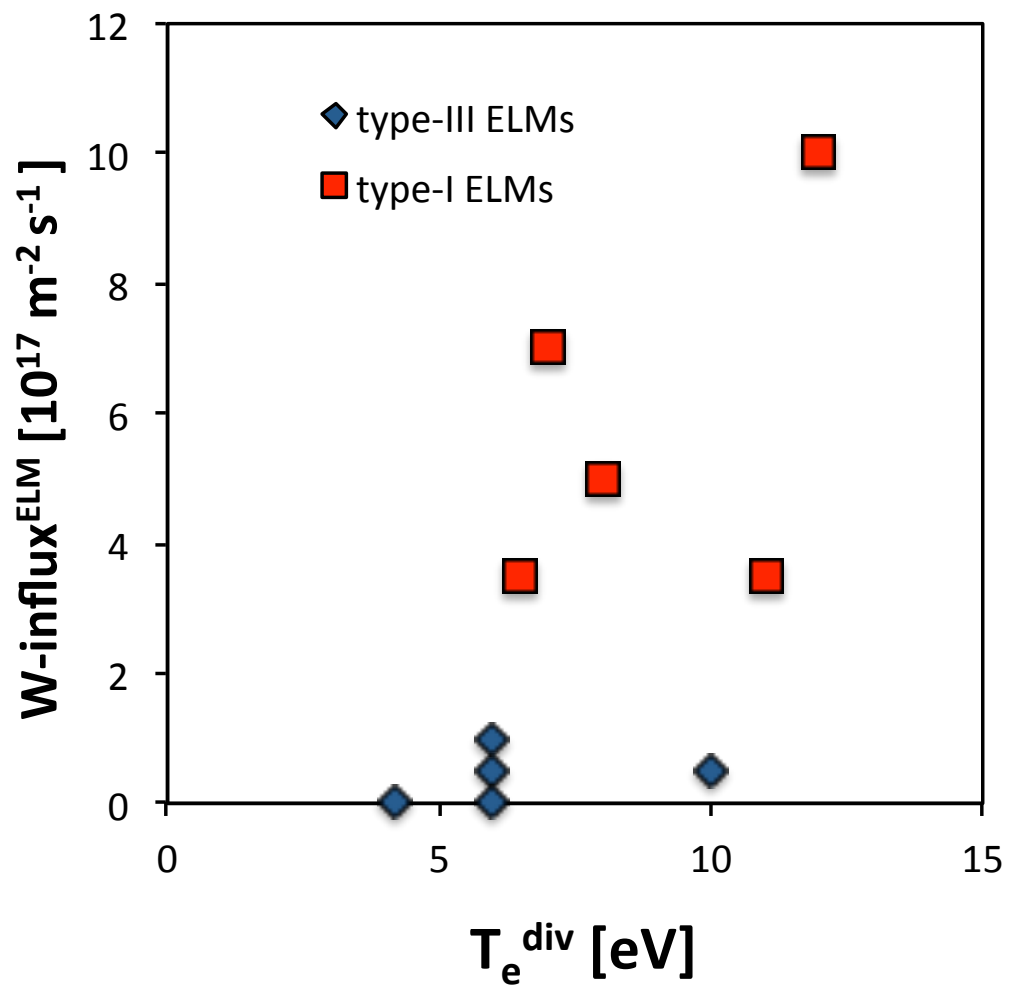




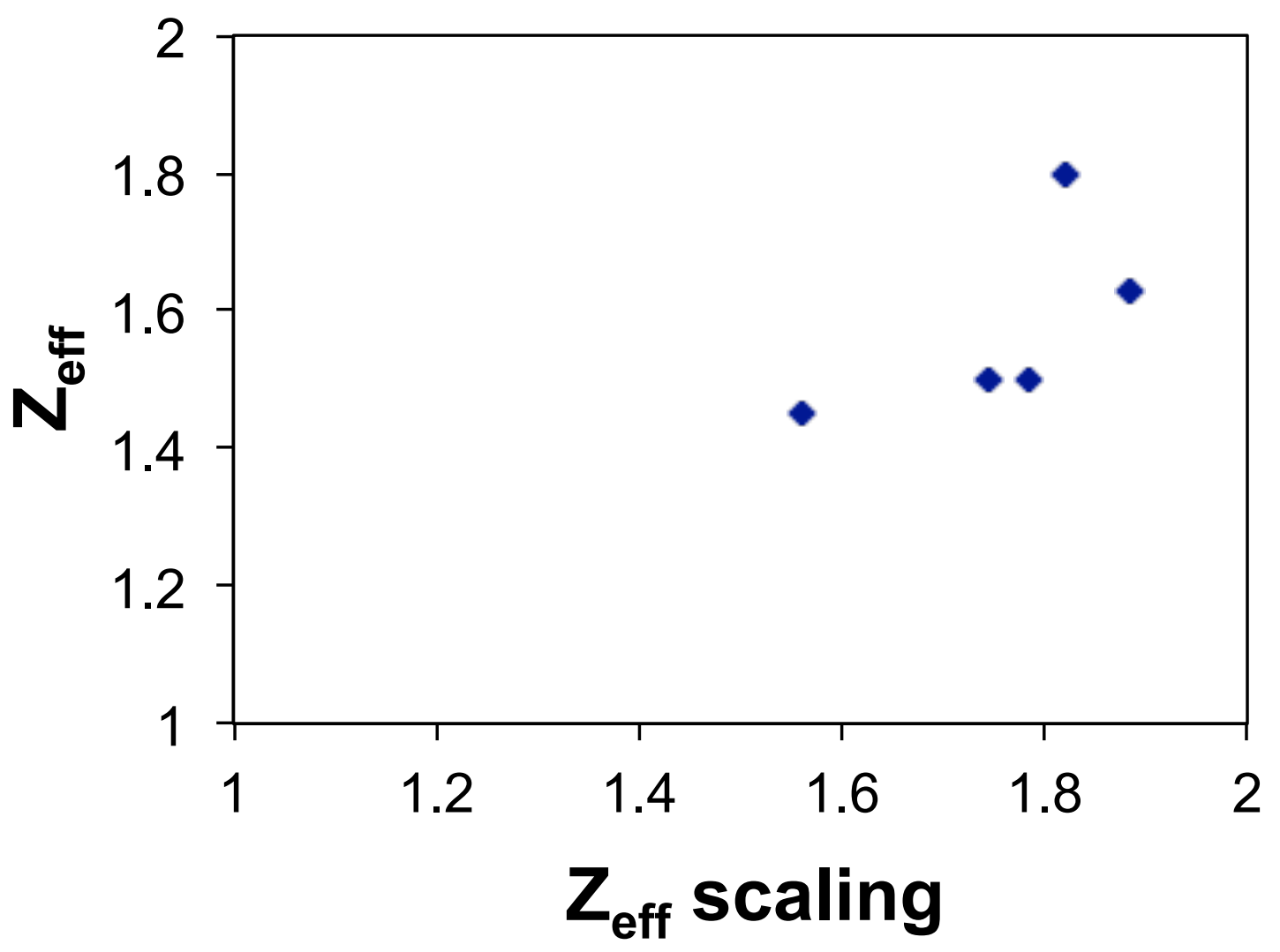

